\title{
Concordance of folate receptor-a expression between biopsy, primary tumor and metastasis in breast cancer and lung cancer patients
}

\author{
Leonora S.F. Boogerd ${ }^{1, *}$, Martin C. Boonstra ${ }^{1, *}$, Ann-Jean Beck ${ }^{1}$, Ayoub Charehbili ${ }^{1}$, \\ Charlotte E.S. Hoogstins ${ }^{1}$, Hendrica A.J.M. Prevoo ${ }^{1}$, Sunil Singhal ${ }^{2}$, Philip S. Low ${ }^{3}$, \\ Cornelis J.H. van de Velde $^{1}$ and Alexander L. Vahrmeijer ${ }^{1}$ \\ ${ }^{1}$ Department of Surgery, Leiden Univeristy Medical Center, Leiden, The Netherlands \\ ${ }^{2}$ Department of Thoracic Surgery, Hospital of The University of Pennsylvania, Philadelphia, PA, USA \\ ${ }^{3}$ Department of Chemistry and Center for Drug Discovery, Purdue University, West Lafayette, IN, USA \\ * These authors have contributed equally to this work \\ Correspondence to: Alexander L. Vahrmeijer, email: a.I.vahrmeijer@lumc.nl \\ Keywords: diagnosis, personalized medicine, targeting, oncology, biomarker, Pathology Section \\ Received: August 05, $2015 \quad$ Accepted: January 31, $2016 \quad$ Published: March 02, 2016
}

\section{ABSTRACT}

Folate receptor alpha ( $F R a)$ is known to be upregulated in a variety of cancers, including non-small cell lung cancer (NSCLC) and breast cancer. To ensure reliable implementation of diagnostic- and therapeutic agents, concordance of FRa expression between biopsy, primary tumor and metastases is important. Using immunohistochemistry (Mab 26B3.F2) these concordances were investigated in 60 NSCLC and $\mathbf{4 0}$ breast cancer patients. False positivity of FRa expression on breast and lung cancer biopsies was limited to less than 5\%. In NSCLC, FRa expression was shown in 21/34 adenocarcinomas and 4/26 squamous cell carcinomas (SCC). Concordance of FRa expression between biopsy and primary tumor was achieved in respectively $83 \%$ and $91 \%$ of adenocarcinomas and SCCs. Approximately $80 \%$ of all local and distant metastases of NSCLC patients showed concordant FRa expression as their corresponding primary tumor. In breast cancer, FRa positivity was shown in 12/40 biopsies, 20/40 lumpectomies and 6/20 LN metastases, with concordance of $68 \%$ between biopsy and primary tumor and $60 \%$ between primary tumor and LN metastases. In conclusion, this study shows high concordance rates of $F R a$ expression between biopsies and metastases compared to primary NSCLC and breast cancers, underscoring the applicability of FRa-targeted agents in these patients.

\section{INTRODUCTION}

The introduction of molecular targeted oncologic therapies has created the need to select patients with the right expression patterns to achieve optimal results [1]. Currently, the molecular markup of tumors can be determined using (real-time or quantitative reverse transcription) polymerase chain reaction, microRNA detection, or immunohistochemistry(IHC). Immunohistochemical staining of tumor tissue is the preferred method, since it provides insight about the expression pattern of specific membrane-bound proteins and, in addition, is a relatively easy and inexpensive technique. Immunohistochemical staining of tumor biopsies is already implemented in clinical practice and used as an efficient selection method in cancer care in, for example, breast cancer patients [2]. Concordance of biomarker expression between biopsy, primary tumor and (distant) metastasis is important for appropriate application of tumor-targeted modalities; e.g. Positron Emission Tomography (PET) or Single Photon Emission Computed Tomography (SPECT)-targeted agents or targeted therapies. For example, a biopsy can turn out to be positive for a certain biomarker while the distant metastasis showed to be negative in most of the cases, resulting in suboptimal applications/results of the targeted approaches. In this study, we investigate these concordances in NSCLC and breast cancer patients and generate insight for the reliable application of FR $\alpha$ targeted agents in these patients. 
In the past decades, several membrane-located proteins were discovered that could successfully be targeted, i.e. estrogen/progesterone receptor (ER/PR) in breast cancer patients and epidermal growth factor receptor (EGFR) in lung cancer patients [2, 3]. Another potential target that holds great promise is folate receptor alpha $(F R \alpha)$. This glycosylphosphatidylinositol (GPI)anchored cell membrane protein was first described on a human ovarian cancer cell line in 1991 and is part of the folate receptor (FR) family [4, 5]. Folic acid and 5-methyltetrahydrofolate, the major circulating form of folate, bind with high affinity to these FRs [6]. Folate, the water soluble vitamin B9, is involved in one-carbon metabolic reactions and is therefore required in the biosynthesis of the nucleotide bases thymine and uracil. The essential role of folate in growth, proliferation and survival of cells reflects the importance of folate during growth and sustenance of tumor cells $[7,8]$. In total, four isoforms of FR are described of which FR $\beta$ and FR $\gamma$ are sited on hematopoietic cells and overexpressed on cancer cells of hematopoietic origin [7]. The isoform FR $\beta$ is also found on activated macrophages, while the function of $\mathrm{FR} \delta$ remains unclear. FR $\alpha$ is normally located on the apical surface of a restricted number of healthy epithelial tissues, such as proximal kidney tubules, fallopian tube and type I and II pneumocytes of the lung [9]. Research on FR $\alpha$ has focused on identification of tumor types that show FR $\alpha$ overexpression and a recent study showed that $40 \%$ of epithelial tumors show FR $\alpha$ overexpression, including (non-mucinous) ovarian, kidney, uterine, colon, lung and breast cancer [10].

Lung cancer is the number one cause of cancer death worldwide with a poor five-year survival estimated at $15 \%$. This disease can be divided in NSCLC, contributing to $85 \%$ of all lung cancers, and small cell lung cancer (SCLC). NSCLC is further subdivided by histological subtype, of which adenocarcinoma (40\%), squamous cell carcinoma (SCC) (25-30\%) and large-cell carcinoma $(10-15 \%)$ are the most prevalent [11]. Tumor-targeted therapies have already been extensively studied in lung cancer patients, which resulted in implementation of such therapies, e.g. bevacizumab, erlotinib and gefitinib, in the treatment guideline of lung cancer disease [12]. Overexpression of FR $\alpha$ in lung cancer is mainly restricted to NSCLC and can therefore be an interesting target for many lung cancer patients [13, 14]. In breast cancer, treatment is already personalized by molecular protein expression of the tumor [2]. The outcome of breast cancer patients is significantly improved by targeted treatment focused on hormone receptor (HR) positive, or human epidermal growth factor receptor 2 (HER2) positive tumors. However, $15 \%$ of all breast cancers show no expression of either ER/PR or HER2 and are considered triple negative (TN) breast cancers. This type of breast cancer has the worst prognosis amongst all breast cancers [15]. Previous studies reported FR $\alpha$ overexpression on especially the majority of TN breast cancers and therefore, FR $\alpha$ could be an interesting target for this specific group of patients [16].

The aim of the present study is to determine $\operatorname{FR} \alpha$ expression on both NSCLC and breast cancers and to determine concordance between FR $\alpha$ expression on biopsy, primary tumor and corresponding local and distant metastatic tissue.

\section{RESULTS}

\section{Patient- and tumor characteristics}

Patient- and histopathological characteristics of all NSCLC and breast cancer patients are summarized in Table 1. In short, mean age of breast and NSCLC patients

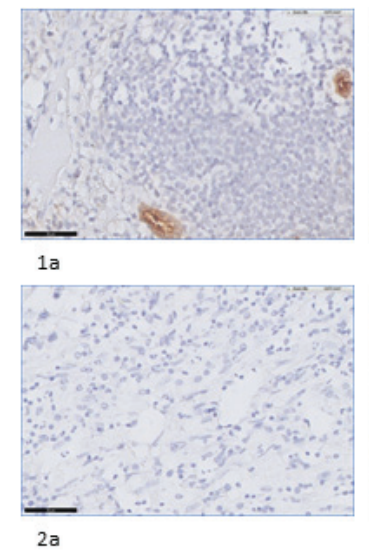

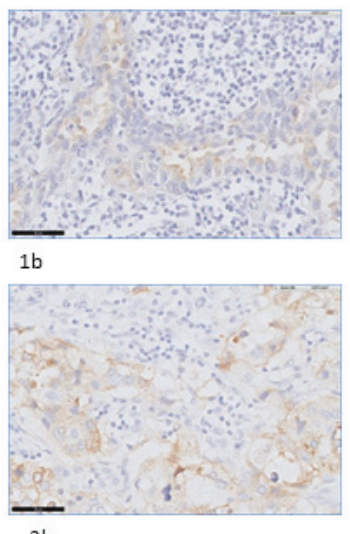

$2 b$

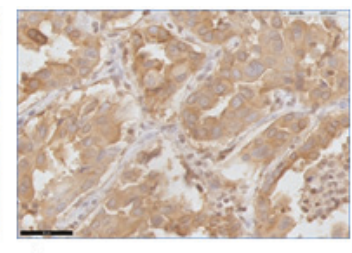

$1 \mathrm{c}$

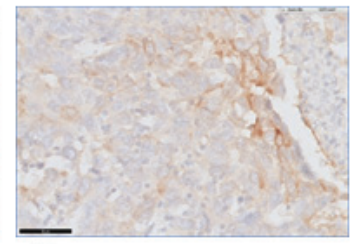

$2 c$

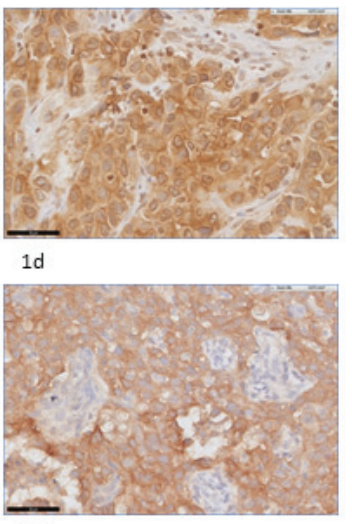

$2 d$

Figure 1: Staining intensities of FR $\alpha$ in NSCLC and breast cancer samples using immunohistochemistry (IHC). The staining score $(0$ to $3+)$ was obtained by assessment of membrane intensity. A score of 0 equals no membrane staining, a score of $1+$ a faint apical membrane staining, a score of $2+$ a moderate apical and occasional lateral membrane staining and a score of $3+$ equals a strong circumferential membrane staining. 1a-1d: staining intensity of respectively $0,1+, 2+$ and $3+$ in adenocarcinoma samples of NSCLC patients (40x) 2a-d: staining intensity of respectively $0,1+, 2+$ and $3+$ in breast cancer samples (40x) 
Table 1: Patient and tumor characteristics

\begin{tabular}{|c|c|c|c|}
\hline Variable & NSCLC, adenocarcinoma No. (\%) & NSCLC, SCC No. (\%) & Breast cancer No. (\%) \\
\hline All patients & $34(57)$ & $26(43)$ & 40 \\
\hline $\begin{array}{l}\text { Mean age at diagnosis } \\
\text { (years) }\end{array}$ & 64.5 & 62.5 & 56 \\
\hline \multicolumn{4}{|l|}{ Sex } \\
\hline male & $21(87.5)$ & $20(77)$ & $0(0)$ \\
\hline female & $13(12.5)$ & $6(23)$ & $40(100)$ \\
\hline \multicolumn{4}{|l|}{ Tumor size } \\
\hline pT1 & $10(29)$ & $3(12)$ & $23(57.5)$ \\
\hline pT2 & $5(15)$ & $6(23)$ & $16(40)$ \\
\hline pT3 & $4(12)$ & $4(15)$ & $1(2.5)$ \\
\hline pT4 & $15(44)$ & $13(50)$ & $0(0)$ \\
\hline \multicolumn{4}{|l|}{ Nodal Stage } \\
\hline $\mathrm{Nx}$ & $0(0)$ & $0(0)$ & $1(2.5)$ \\
\hline N0 & $7(21)$ & $5(19)$ & $12(30)$ \\
\hline N1-2 & $27(79)$ & $21(81)$ & $27(67.5)$ \\
\hline \multicolumn{4}{|l|}{ IASCL stage } \\
\hline IA & $4(12)$ & $1(4)$ & \\
\hline IB & $2(6)$ & $2(8)$ & \\
\hline IIA & $3(9)$ & $4(15)$ & \\
\hline IIB & $2(6)$ & $0(0)$ & \\
\hline IIIA & $7(20)$ & $10(38)$ & \\
\hline IIIB & $1(3)$ & $1(4)$ & \\
\hline IV & $15(44)$ & $8(31)$ & \\
\hline \multicolumn{4}{|l|}{ Marker status } \\
\hline \multicolumn{4}{|l|}{ HR status } \\
\hline positive & & & $19(47.5)$ \\
\hline negative & & & $21(52.5)$ \\
\hline \multicolumn{4}{|l|}{ HER2 status } \\
\hline positive & & & $17(42.5)$ \\
\hline negative & & & $23(57.5)$ \\
\hline \multicolumn{4}{|l|}{$\mathrm{TN}$} \\
\hline yes & & & $15(37.5)$ \\
\hline no & & & $25(62.5)$ \\
\hline \multicolumn{4}{|l|}{ Histology } \\
\hline Ductal & & & $36(90)$ \\
\hline Lobular & & & $4(10)$ \\
\hline \multicolumn{4}{|l|}{ Tumor grade } \\
\hline $\mathrm{NE}$ & & & $4(10)$ \\
\hline Grade 1 & & & $2(5)$ \\
\hline Grade 2 & & & $13(32.5)$ \\
\hline Grade 3 & & & $21(52.5)$ \\
\hline \multicolumn{4}{|l|}{ Neo-adjuvant therapy } \\
\hline Yes & $14(41)$ & $8(31)$ & $6(15)$ \\
\hline No & $20(59)$ & $18(69)$ & $34(85)$ \\
\hline
\end{tabular}

Abbreviations: NSCLC non-small cell lung cancer; SCC squamous cell carcinoma; pT pathological tumor stage, N node status, IASCL International Association for the Study of Lung Cancer; HR hormone receptor, including progesterone and estrogen receptor status; Her2 human epidermal growth factor receptor 2; NE not evaluable; Tumor grade, according to Bloom \& Richardson scoring method 
Table 2: Folate receptor- $\alpha$ expression in NSCLC

\begin{tabular}{|c|c|c|c|c|}
\hline & Total No. & $\operatorname{FR} \alpha(+)$ No. $(\%)$ & $\operatorname{FR} \alpha(-)$ No. (\%) & $P$-value \\
\hline \multicolumn{5}{|l|}{ Biopsies } \\
\hline Total & 23 & & & \\
\hline Adenocarcinoma & 12 & $8(67)$ & $4(33)$ & 0.036 \\
\hline $\mathrm{SCC}$ & 11 & $2(18)$ & $9(82)$ & \\
\hline \multicolumn{5}{|l|}{ Primary tumor } \\
\hline Total & 60 & & & \\
\hline Adenocarcinoma & 34 & $21(62)$ & $13(38)$ & $<0.001$ \\
\hline $\mathrm{SCC}$ & 26 & $4(15)$ & $22(85)$ & \\
\hline \multicolumn{5}{|c|}{ Lymph node metastases } \\
\hline Total & 60 (in 33 pts) & & & \\
\hline Adenocarcinoma & 42 (in 18 pts) & $26(62)$ & $16(38)$ & 0.004 \\
\hline $\mathrm{SCC}$ & 18 (in 15 pts) & $3(17)$ & $15(83)$ & \\
\hline \multicolumn{5}{|l|}{ Distant metastasis } \\
\hline Total & 23 & & & \\
\hline Adenocarcinoma & 15 & $5(33)$ & $10(67)$ & 0.122 \\
\hline $\mathrm{SCC}$ & 8 & $0(0)$ & $8(100)$ & \\
\hline
\end{tabular}

Abbreviations: SCC squamous cell carcinoma; FR $\alpha$ Folate receptor $\alpha$; pts patients

was respectively 56 and 63.5 years at diagnosis. The majority of breast cancer patients displayed pT1 or pT2 stage tumors and all of them underwent a lumpectomy. The majority of NSCLC patients displayed pT4 tumors and IASCL stage IV. Of both NSCLC and breast cancer patients approximately $70 \%$ showed lymph node involvement, i.e. nodal stage N1-2. The cohort of breast cancer patients consisted of $15 \mathrm{TN}$ breast cancers with a majority of high grade tumors, e.g. 21 out of 40 tumors showed differentiation grade III.

\section{FR $\alpha$ expression and concordance between biopsy, primary tumor and metastases in NSCLC patients}

Of all primary tumors containing adenocarcinoma $(N=34), 21$ out of 34 tumors showed FR $\alpha$ expression of which the majority $(>80 \%)$ showed overexpression (Figure 1, Table 2). Heterogeneity of FR $\alpha$ expression was seen in 16 out of $21 \mathrm{FR} \alpha$-expressing adenocarcinomas. Of all primary tumors containing $\operatorname{SCC}(N=26)$, only 4 out of 26 tumors showed FR $\alpha$ expression. Overexpression was seen in $1 \mathrm{SCC}$ and a heterogeneous staining pattern in 3 out of 4 SCCs. Of all biopsy specimens $(N=23), 8$ out of 12 adenocarcinomas showed FR $\alpha$ expression whereas only 2 out of 11 SCCs showed FR $\alpha$ expression. Of all 60 metastatic LNs, obtained from 33 patients, 26 out of 42 LNs containing adenocarcinoma showed FR $\alpha$ expression, whereas 3 out of 18 LNs containing SCC showed FR $\alpha$ expression. Of all distant metastases $(N=23), \mathrm{FR} \alpha$ expression was shown in 5 out of 15 adenocarcinomas but in none of the 8 SCCs.

Concordance of FR $\alpha$ expression between biopsy and primary tumor was shown in 20 out of 23 biopsies (Figure 2, Table 3). Two of the disconcordances (one adenocarcinoma and one SCC) were attributed to loss of $\operatorname{FR} \alpha$ expression in biopsy specimens, while primary tumors did show expression. The other disconcordance was due to upregulation of FR $\alpha$ expression on the biopsy specimen, containing adenocarcinoma. In conclusion, only one biopsy specimen showed false positivity. Concordance between local metastasis, e.g. metastatic LNs, and primary tumor was shown in 31 out of 42 LNs containing adenocarcinoma, within 14 of 18 patients. All disconcordances could be attributed to loss of FR $\alpha$ expression in metastatic LNs. In SCC, concordance of FR $\alpha$ expression between metastatic LNs and primary tumor was achieved in 13 out of 18 LNs, within 12 of 15 patients. In $3 \mathrm{LNs}$ disconcordance was attributed to upregulation of FR $\alpha$ expression, while 2 LNs showed downregulation compared to $\mathrm{FR} \alpha$ expression in the primary tumor. Concordance between primary tumors and corresponding distant metastases was seen in 12 out of 15 adenocarcinomas and in 7 out of 8 SCC (Figure 3). Disconcordance in 2 of the metastatic adenocarcinomas and in the metastasis that contained SCC was attributed to downregulation of the distant metastases compared to the primary tumor. The other bone metastasis containing adenocarcinoma showed upregulation compared to the corresponding primary tumor. 


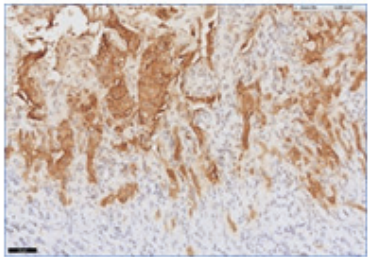

$1 \mathrm{a}$

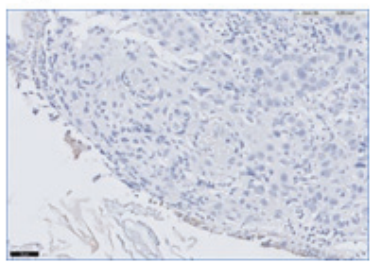

$2 a$

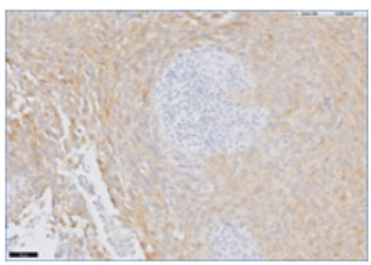

$3 a$

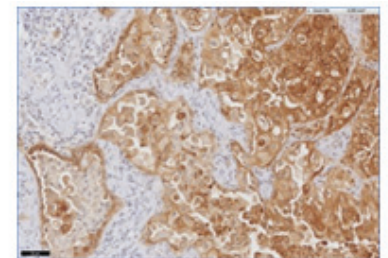

$1 b$

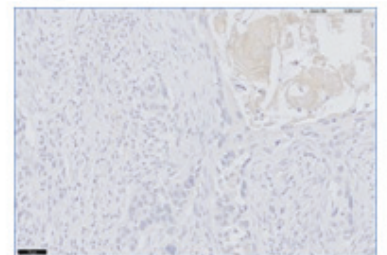

$2 b$

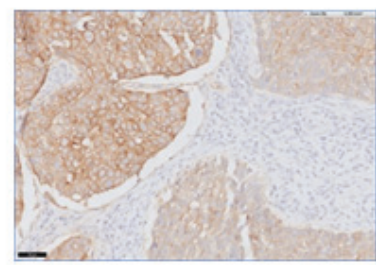

$3 b$

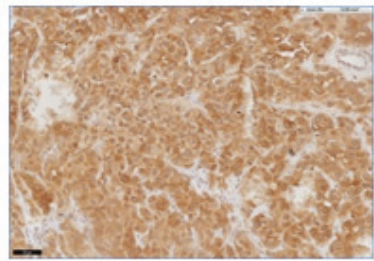

$1 c$

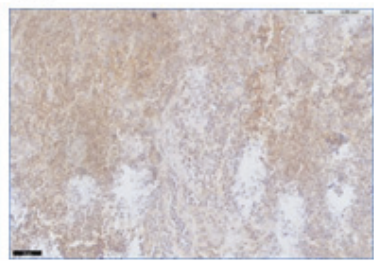

$2 c$

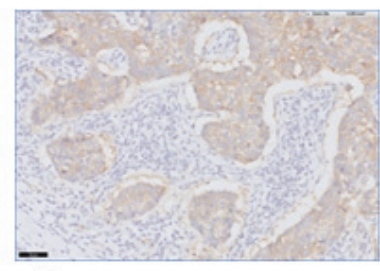

$3 c$

Figure 2: Examples of (dis)concordance in FRa staining in biopsy-, primary tumor-, and metastatic LN tissue in NSCLC and breast cancer patients. 1a-1c: example of concordance between positive FR $\alpha$ expression on biopsy (1a), primary tumor (1b) and metastatic LN tissue (1c) in a NSCLC patient, containing adenocarcinoma (20x). 2a-2c: example of disconcordance between FR $\alpha$ expression on biopsy (2a), primary tumor (2b) and metastatic LN tissue (2c) in a NSCLC patient, containing SCC (20x). 3a-3c: example of concordance between positive FR $\alpha$ expression on biopsy (3a), primary tumor (3b) and metastatic LN tissue (3c) in a breast cancer patient (20x).

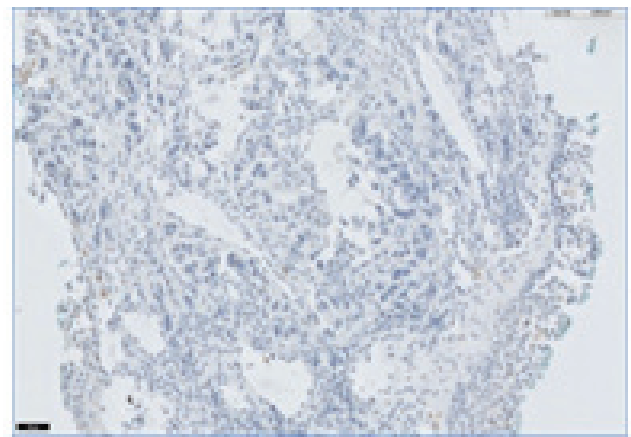

$1 \mathrm{a}$

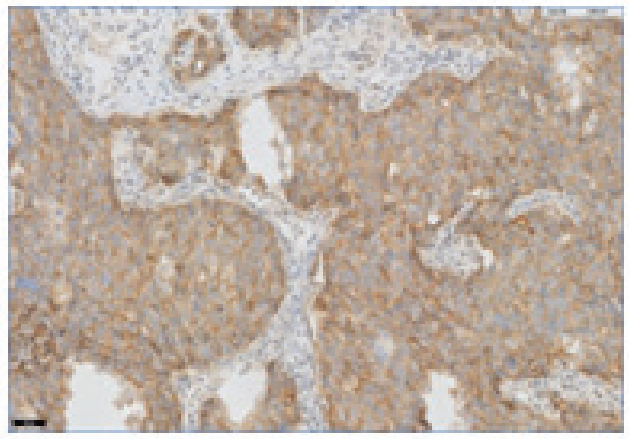

$2 a$

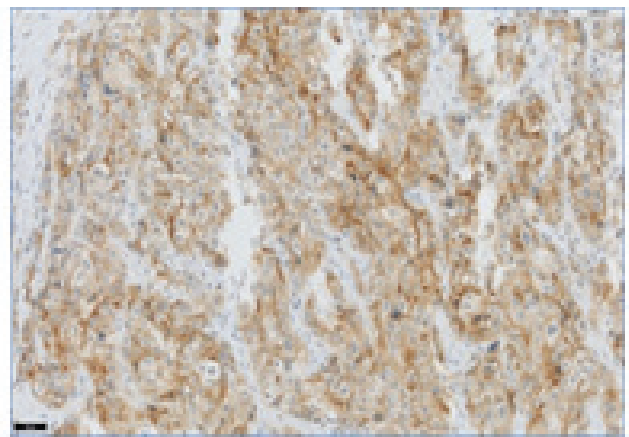

1b

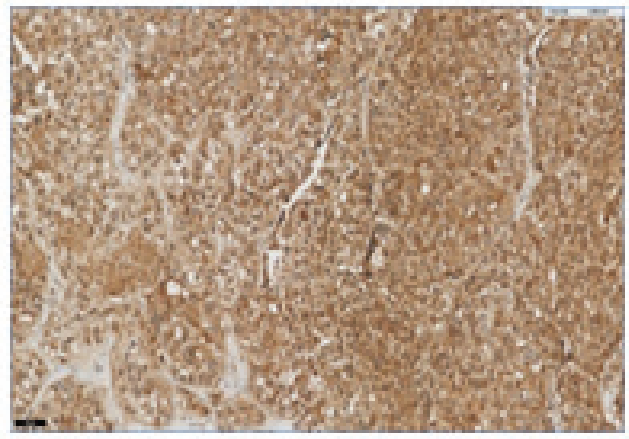

$2 b$

Figure 3: FR $\alpha$ expression in NSCLC and corresponding distant metastases. 1a: primary tumor containing adenocarcinoma without FR $\alpha$ expression (20x). 1b: corresponding distant metastasis of the bone with positive FR $\alpha$ expression (20x). 2a: primary tumor containing adenocarcinoma with positive FR $\alpha$ expression (20x). 2b: corresponding distant metastasis of the brain with positive FR $\alpha$ expression (20x). 
Table 3: Concordance between biopsy, primary tumor and corresponding disseminated lymph nodes and distant metastases in patients with breast cancer and NSCLC

\begin{tabular}{|c|c|c|c|}
\hline Degree of concordance & $\begin{array}{l}\text { Breast cancer patients } \\
\text { No. }(\%)\end{array}$ & $\begin{array}{l}\text { NSCLC } \quad \text { patients, } \\
\text { adenocarcinoma No. }(\%)\end{array}$ & $\begin{array}{l}\text { NSCLC patients, } \\
\text { SCC, No. }(\%)\end{array}$ \\
\hline \multicolumn{4}{|l|}{ Concordance of FR $\alpha$ status } \\
\hline Biopsy $=$ Primary tumor & 27 out of $40(67.5)$ & 10 out of $12(83)$ & 10 out of $11(91)$ \\
\hline Primary tumor $=$ Local metastasis & 12 out of $20(60)$ & 31 out of $42(78)^{*}$ & 13 out of $18(80)^{*}$ \\
\hline Primary tumor $=$ Distant metastases & & 12 out of $15(80)$ & 7 out of $8(88)$ \\
\hline \multicolumn{4}{|l|}{ Disconcordance of FR $\alpha$ status } \\
\hline Biopsy $>$ Primary tumor & 2 & 1 & 0 \\
\hline$<$ Primary tumor & 11 & 1 & 1 \\
\hline Primary tumor $>$ Local metastasis & 6 & 4 & 2 \\
\hline Primary tumor $<$ Local metastasis & 2 & 0 & 3 \\
\hline Primary tumor $>$ Distant metastasis & & 2 & 1 \\
\hline Primary tumor $<$ Distant metastasis & & 1 & 0 \\
\hline
\end{tabular}

Abbreviations: SCC squamous cell carcinoma; FR $\alpha$ Folate receptor $\alpha$; pos LN positive lymph node * LN expression was assessed in 33 patients, in respectively 18 patients with adenocarcinoma and 15 patients with SCC; concordance was seen in 14 out of 18 adenocarcinoma patients (31/42 LNs) and 12 out of 15 SCC patients (13/18 LNs).

\section{FR $\alpha$ expression and concordance between biopsy, primary tumor and metastases in breast cancer patients}

Of the total cohort of breast cancer patients in this study $(N=40)$, a positive $\mathrm{FR} \alpha$ expression was seen in 12 of the 40 biopsy specimens, 20 of the 40 lumpectomy specimens and 6 of the 20 metastatic LNs (Figure 1, Table 4). Overexpression of FR $\alpha$ was seen in the majority of biopsies, lumpectomy specimens and metastatic LNs, however, almost no homogenous staining patterns were detected. In total, only 5 of the 20 primary tumors showed a homogenous staining pattern. Of all tissue, the hormone receptor (HR) status was known and correlated with FR $\alpha$ expression. As described in Table 4, the HR status, e.g. ER/PR status, showed to correlate negatively with FR $\alpha$ expression of biopsies $(P=0.002)$ and lumpectomy specimens $(P=0.010)$. Of all $15 \mathrm{TN}$ breast cancers that were included in this study, e.g. ER-PR-Her2-, respectively 7 biopsies $(P=0.091)$ and 12 primary tumor specimens $(P=0.008)$ showed a positive FR $\alpha$ expression. In addition, 3 out of 7 metastatic LNs of TN breast cancers showed FR $\alpha$ positivity $(P=0.613)$. Of all biopsy and lumpectomy tissue, a subdivision of FR $\alpha$ expression per LN status was made. Of all specimens of patients with LN metastases $(N=27), 7$ out of 27 biopsies showed a positive FR $\alpha$ expression and 12 out of 27 lumpectomy specimens.

Concordance of FR $\alpha$ expression between biopsy and primary tumor was shown in 27 out of 40 specimens (Figure 2, Table 3). Disconcordance was attributed to downregulation of FR $\alpha$ on the biopsy specimens compared to the primary tumor in 11 of the 13 tissue sections and upregulation in 2 tissue sections. In conclusion, based on concordance of biopsy and primary tumor tissue, only 2 of the 40 evaluated tissue specimens showed false positivity. Furthermore, in $50 \%$ of all tissues, a similar FR $\alpha$ expression was shown in biopsy, lumpectomy and metastatic LN. Concordance between primary tumor and metastatic LN, in the cohort with LN metastases, was seen in 12 out of 20 specimens. Subanalysis showed that 6 out of these 8 disconcordances were attributed to downregulation of $\mathrm{FR} \alpha$ expression on metastatic LNs compared to the primary tumor. The remaining two LNs showed a positive FR $\alpha$ expression, whereas the corresponding lumpectomy specimen did not show FR $\alpha$ expression. Separate analysis of the 6 patients that received neoadjuvant therapy showed in 4 out of these 6 patients concordant FR $\alpha$ expression between primary tumor and biopsy specimen. All these 4 patients showed no FR $\alpha$ expression in biopsy nor in primary tumor. The other two patients both showed a positive FR $\alpha$ expression on the primary tumor, but no expression on corresponding biopsy. In 5 of the 6 patients who received neoadjuvant therapy, metastatic LNs were available for evaluation of FR $\alpha$ expression. Only one metastatic LN showed FR $\alpha$ positivity, which was not concordant with the absent FR $\alpha$ expression of the primary tumor.

\section{DISCUSSION}

Since $\operatorname{FR} \alpha$ has emerged as target for imaging and treatment purposes, several clinical trials have been conducted with promising results $[10,17]$. Diagnostic and therapeutic agents can be delivered intracellular through FR $\alpha$-mediated endocytosis [18]. During this process, an endosome (containing a FR $\alpha$-targeted agent bound to $\mathrm{FR} \alpha$ ) is formed which moves to the recycling center, close to the nucleus. Here, the folate-agent detaches from its 
Table 4: Folate receptor- $\alpha$ expression in breast cancer patients

\begin{tabular}{|c|c|c|c|c|}
\hline Biopsy & Biomarker status & FRa(+) No. (\%) & FRa(-) No. (\%) & $P$-value \\
\hline Total & & 12 & 28 & \\
\hline \multirow[t]{2}{*}{ HR status } & positive & $1(8)$ & $18(64)$ & 0.002 \\
\hline & negative & $11(92)$ & $10(36)$ & \\
\hline \multirow[t]{2}{*}{ HER2 status } & positive & $5(42)$ & $12(43)$ & 1.000 \\
\hline & negative & $7(58)$ & $16(57)$ & \\
\hline \multirow[t]{2}{*}{$\mathrm{TN}$} & yes & $7(58)$ & $8(29)$ & 0.091 \\
\hline & no & $5(42)$ & $20(72)$ & \\
\hline \multirow[t]{2}{*}{ Node status } & positive & $7(58)$ & $20(71)$ & 0.476 \\
\hline & negative & $5(42)$ & $8(29)$ & \\
\hline \multicolumn{5}{|l|}{$\begin{array}{l}\text { Lumpectomy } \\
\text { specimen }\end{array}$} \\
\hline Total & & 20 & 20 & \\
\hline \multirow[t]{2}{*}{ HR status } & positive & $5(25)$ & $6(30)$ & 0.010 \\
\hline & negative & $15(75)$ & $14(70)$ & \\
\hline \multirow[t]{2}{*}{ HER2 status } & positive & $7(35)$ & $10(50)$ & 0.523 \\
\hline & negative & $13(65)$ & $10(50)$ & \\
\hline \multirow[t]{2}{*}{$\mathrm{TN}$} & yes & $12(60)$ & $3(15)$ & 0.008 \\
\hline & no & $8(40)$ & $17(85)$ & \\
\hline \multirow[t]{2}{*}{ Node status } & positive & $12(60)$ & $15(75)$ & 0.501 \\
\hline & negative & $8(40)$ & $5(25)$ & \\
\hline \multicolumn{5}{|c|}{\begin{tabular}{ll|l} 
Lymph & node & \\
metastasis & & \\
\end{tabular}} \\
\hline Total & & 6 & 14 & \\
\hline \multirow[t]{2}{*}{ HR status } & positive & $2(33)$ & $7(50)$ & 0.642 \\
\hline & negative & $4(67)$ & $7(50)$ & \\
\hline \multirow[t]{2}{*}{ HER2 status } & positive & $2(33)$ & $8(57)$ & 0.628 \\
\hline & negative & $4(67)$ & $6(43)$ & \\
\hline \multirow[t]{2}{*}{$\mathrm{TN}$} & yes & $3(50)$ & $4(29)$ & 0.613 \\
\hline & no & $3(50)$ & $10(71)$ & \\
\hline
\end{tabular}

Abbreviations: FR $\alpha$ Folate receptor $\alpha$; HR hormone receptor including estrogen- and progesterone receptor; HER2 human epidermal growth factor receptor 2; TN triple negative

receptor and is released, in contrast to the receptor that is brought back to the cell surface. This pathway is mainly responsible for accumulation of FR $\alpha$-targeted agents in malignant cells. However, absorption of folate in normal tissue such as duodenum and jejunum is facilitated by the proton-coupled folate transporter and the reduced folate carrier (RFC). The latter anion exchanger is responsible for transport of the majority of folate in healthy tissues, while folate-coupled agents show no affinity for this RFC nor the proton-coupled folate transporter [7, 19].

As reported by Hilgenbrink and Low et al. [20], folate has been linked to a wide variation of substances used in cancer therapy, including protein toxins, enzymes to activate prodrugs, chemotherapeutics, immunotherapeutic agents, drug-comprising liposomes and nanoparticles. Currently, farletuzumab, vintafolide and IMGN853 are the three FR $\alpha$-targeting agents that show the most potential in clinical cancer trials, among other as treatment strategy for lung cancer $[10,21]$. Detection of malignancies by FR $\alpha$-targeted imaging approaches is studied broadly using folate linked to radionuclides, PET agents, MRI contrast agents and fluorescent dyes. In NSCLC, a recent study of Okusanya et al. showed feasibility of intraoperative detection of small subpleural lung tumors using a fluorescent FR $\alpha$-targeted molecular agent, e.g. folate-FITC (Figure 4) [22]. An excellent sensitivity and specificity was shown, resulting in the start of a novel study with the same targeting ligand but optimized fluorophore, e.g. OTL-38 (clinicaltrial.gov). Analysis of a similar clinical trial in breast cancer patients is currently ongoing to determine tumor detection during breast cancer surgery using folate-FITC (clinicaltrial.gov).

In order to efficiently apply both FR $\alpha$-targeted therapeutic and imaging agents, adequate patient selection regarding $\mathrm{FR} \alpha$ expression is required. In addition, knowledge about the concordance of FR $\alpha$ expression between biopsy, primary tumor specimen and (possible) metastasis is pivotal to estimate efficacy and usability 
of these approaches. Based on results from the current study, selection of breast cancer and NSCLC patients who might benefit from FR $\alpha$-targeted approaches can be performed reliably via biopsy staining. Although the majority of tumor specimens showed heterogeneity of FR $\alpha$ expression, less than $5 \%$ of all biopsies showed false positivity. In a clinical setting, this suggests that 1 out of 20 included breast cancer or NSCLC patients in a $\mathrm{FR} \alpha$-targeted trial would show no $\mathrm{FR} \alpha$ expression in the primary tumor while the biopsy stained FR $\alpha$-positive. Regarding the targeting of disseminated malignant cells, this study shows high concordance rates of local/distant metastases with corresponding primary tumors. Although concordance rates were relatively lower for breast cancer than for NSCLC, e.g. $68 \%$ vs. $>80 \%$, these results still suggest that the majority of metastases can be targeted when the primary tumor shows $\mathrm{FR} \alpha$ expression.

Overall, disconcordance was mainly due to downregulation of FR $\alpha$ in biopsy- and metastastic tissue compared to primary NSCLC and breast cancer, which could be a result of tissue sampling or of heterogeneity of FR $\alpha$ expression. In this study, whole tissue slides were used to overcome the difficulty in evaluating heterogeneity as is experienced with the generally used TMAs [23]. Of all 266 evaluated tumor tissue specimens, heterogeneity of FR $\alpha$ was shown in the majority $(>80 \%)$ of the stained

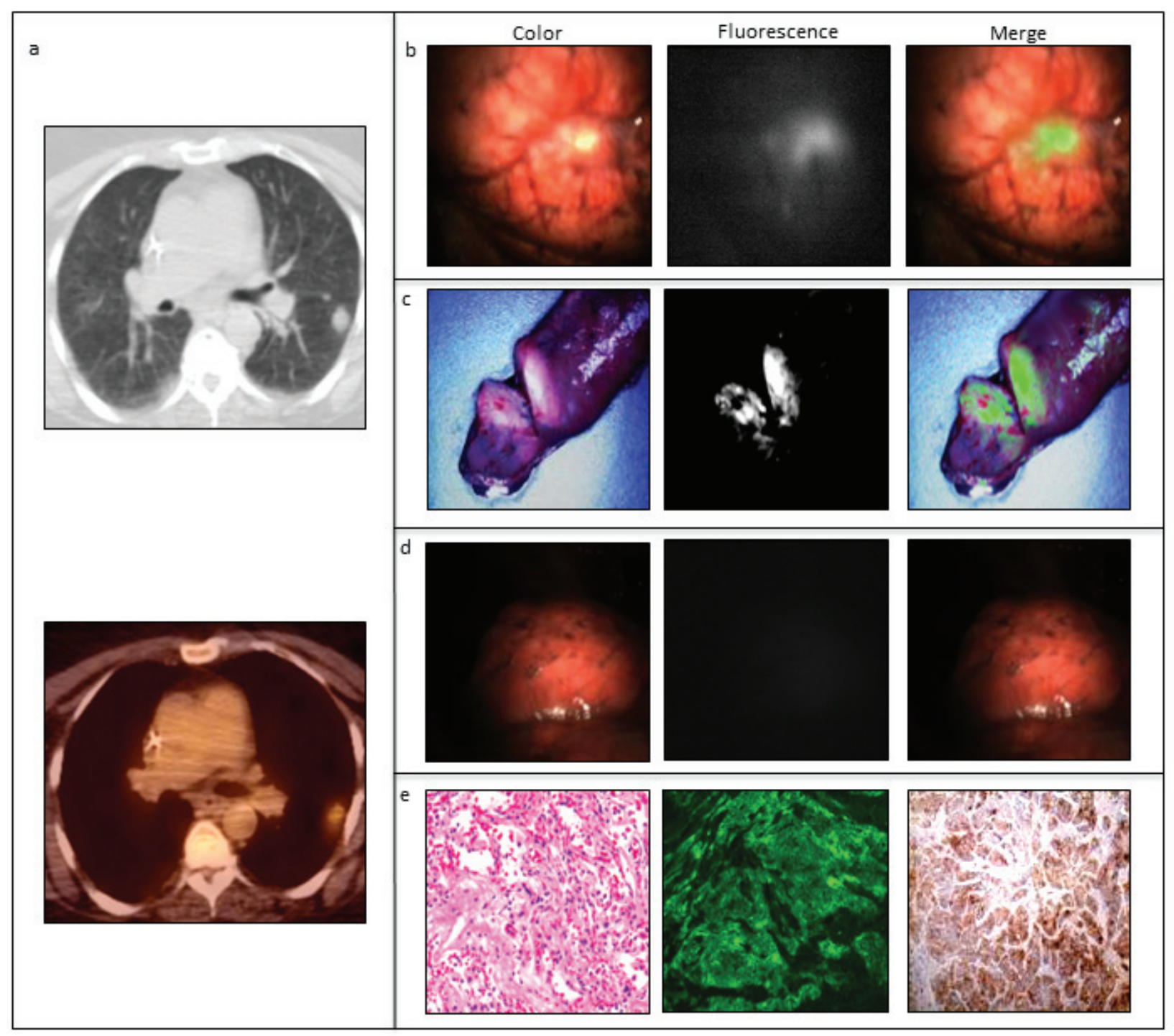

Figure 4: Example of FR $\alpha$-targeted detection of an adenocarcinoma using EC-17, i.e. fluorescent FR $\alpha$-targeted molecular agent, in a patient suffering from NSCLC. In vivo fluorescence imaging was performed using the Artemis imaging system [34]. a: Prior to pulmonary resection, a tumor of $3 \mathrm{~cm}$ in the upper lobe of the left lung is detected by CT-and PET-scan. b: In vivo fluorescence imaging shows clear tumor delineation. c: Ex vivo fluorescence imaging of the tumor in the resected specimen. d: After resection, the wound bed was inspected with $\lambda$ ex $490 \mathrm{~nm}$ and demonstrated no residual fluorescence at the surgical margins. e: FR $\alpha$ upregulation was confirmed by fluorescence microscopy and immunohistochemical staining. 
sections.

Several IHC studies investigated FR $\alpha$ expression on primary lung (NSCLC) and breast cancers. Two lung studies applied the same monoclonal antibody as used in this study, i.e. 26B3.F2, and displayed similar expression rates, underscoring the validity of the used staining: more than $70 \%$ of adenocarcinomas expressed FR $\alpha$ and less than $15 \%$ of SCCs $[14,24]$. In the current study, a significant association between FR $\alpha$ expression and histological subtype was shown in biopsies $(P=0.036)$, primary tumors $(P<0.001)$ and metastatic LNs $(P=0.004)$. The distinction in FRa expression between adenocarcinoma and SCC is caused by the type of cancer cell they derive from; adenocarcinoma originates from FR $\alpha$-expressing type 1 and 2 pneumocytes and SCC from more centrally

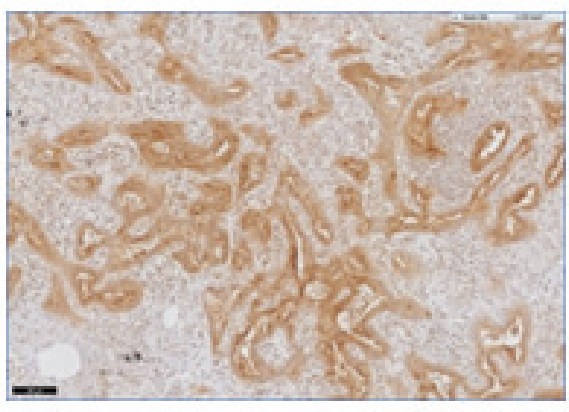

a
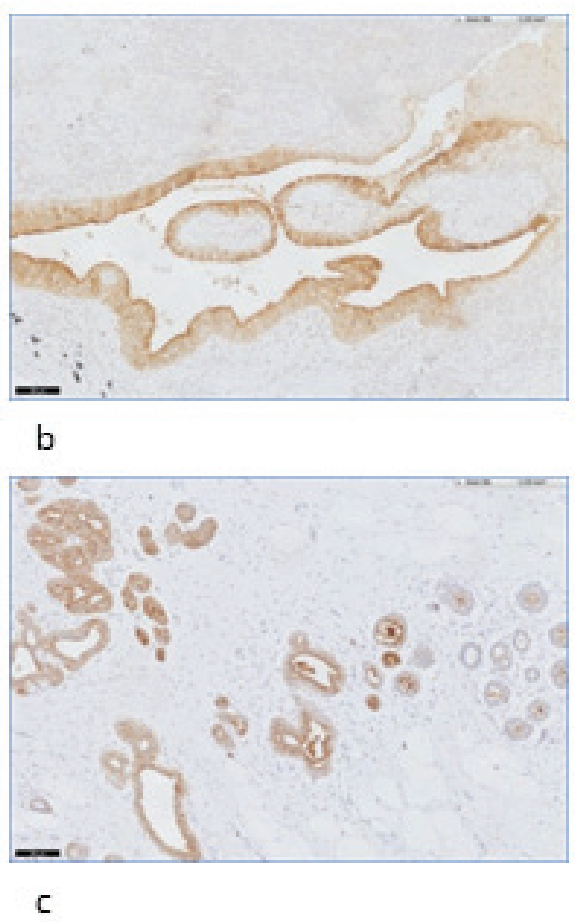

Figure 5: Examples of $F R \alpha$ staining in normal lung and breast tissue. a: staining of $\mathrm{FR} \alpha$ at the luminal border of normal lung tissue in an adenocarcinoma patient (10x). $\mathbf{b}$ : staining of FR $\alpha$ at the luminal border of normal lung tissue in a SCC patient (10x). $\mathbf{c}$ : staining of FR $\alpha$ in normal breast tissue: staining at the luminal border of secretory cells (10x). positioned tracheal cells which do not express the receptor $[14,25]$

Other IHC studies using a different monoclonal antibody (mAB343) showed expression rates in adenocarcinoma and SCC ranging from $72 \%$ to $87 \%$ and $15 \%$ to $57 \%$ respectively $[13,26]$. The difference in percentages of positive FR $\alpha$ expressing tumors between studies may be partially contributed to a variation in applied antibodies. For example, relatively high percentages of FR $\alpha$ expression in patients with SCC obtained using mAB343 may be a result of non-specific binding $[13,26]$. Furthermore, cut-off values for defining FR $\alpha$ positivity and scoring methods are diverse amongst studies. Nunez et al. [13] examined FR $\alpha$ expression in NSCLC patients $(n=320)$ by using the H-score, which offers a separate score for both cytoplasmic and membranous staining. In breast cancer, cut-off values for defining FR $\alpha$ positivity differed from $5-15 \%$. Scoring using the M-score has been described in which both membranous tumor cell staining and the proportion and staining intensity of FR $\alpha$ positive cells are captured [16]. Hartmann et al. [27] assessed FR $\alpha$ expression using mAB343 on a tissue micro array (TMA) containing samples of 63 invasive breast cancers with either poor or good outcome. An association between strong FR $\alpha$ expression and a poor outcome, defined as median time to recurrence of 1.9 years, was shown. The latter results are in line with other studies describing a significant association between FR $\alpha$ expression and worse (diseasefree) survival $[16,28]$. Increased folate storage in FR $\alpha$ expressing tumors may cause accelerated growth and consequently, a more aggressive tumor. However, a recent study describing FR $\alpha$ expression in breast cancer brain metastases was unable to show any association between FR $\alpha$ expression and survival, possibly due to small sample size [29]. In lung cancer, results from studies investigating survival and FR $\alpha$ expression also vary. Several IHC studies and a study investigating FR $\alpha$ gene expression using RT-PCR reported an improved overall survival when FR $\alpha$ expression is high $[14,30]$. However, Nunez et al. could not show any correlation between FR $\alpha$ expression and overall- or recurrence free survival in a cohort of 320 patients [13]. It still remains unclear why high levels of FR $\alpha$ are correlated with a favorable prognosis; further research is warranted to explain this.

Recent studies showed that FR $\alpha$ expressing breast cancers represented a novel molecular subtype associated with ER-/PR-/Her2Neu- and ER- breast cancers [16, 28]. The current study endorses these conclusions as 12 out of 15 primary tumors of TN breast cancers showed a positive FR $\alpha$ expression and a significant correlation between HR status and FR $\alpha$ expression on both biopsy specimens $(P=0.002)$ and primary tumors $(P=0.010)$ was found. It is known that estrogens are involved in the regulation of FR $\alpha$ expression and $17 \beta$ estradiol via the $\mathrm{P} 4$ promoter, resulting in down-regulation of $F R \alpha[31,32]$. 
Positive FR $\alpha$ expression was shown in respectively $30 \%$, $50 \%$ and $30 \%$ of biopsy-, lumpectomy- and metastatic LN tissue. Although this percentage is higher than described in literature, it must be noted that $67.5 \%$ of the included patients had LN metastases (N1-2) and 37.5\% were TN breast cancer patients. Furthermore, tumors displayed a relatively high tumor grade. In the study of Zhang et al. [28], a significant association between FR $\alpha$ positive expression and high histologic grade, high nodal stage and subgroups of ER/PR- and TN breast cancers was shown. In an extra cohort of metastatic stage IV, Her2 negative breast cancer patients, O'Shannessy et al. [16] described that the percentage of $\mathrm{FR} \alpha$ positive tumors in early stage disease is retained in late stage metastatic disease, as $36 \%$ of samples showed FR $\alpha$ positive expression of whom $86 \%$ were TN breast cancers.

In an additional cohort of NSCLC patients $(N=23)$, concordance of FR $\alpha$ expression between primary tumor and corresponding distant metastases was assessed. In the literature, little is known about FR $\alpha$ expression on distant metastases. In a study of O'Shannessy et al. [14], 9 fine needle aspirates comprising LN metastases of lung adenocarcinoma showed $63 \% \mathrm{FR} \alpha$ positivity. Furthermore, Nunez et al. [13] reported no significant difference in FR $\alpha$ expression between 27 NSCLC and 15 derived metastatic sites. We show concordance rates between primary tumor and distant metastases of respectively $80 \%$ and $88 \%$ for adenocarcinomas and SCCs. In addition, all metastatic LNs showed concordant FR $\alpha$ expression with the corresponding distant metastases. On the contrary, Kikuchi et al. [33] showed downregulation of FR $\alpha$ gene expression in 16 metastatic brain tumors compared to 22 primary lung adenocarcinomas using cDNA microarray. However, as this study investigated gene expression and non-corresponding metastases, results have to be interpreted with caution.

Finally, normal breast and lung cells showed FR $\alpha$ expression (Figure 5). In particular the luminal border of secretory cells (due to the secretion of folate in milk) and the myoepithelial layers of normal epithelial breast tissue stained positive. Nonetheless, FR $\alpha$ expression on normal breast and lung tissue will not significantly interfere when FR $\alpha$-targeted agents are intravenously administered, since the receptor is confined to the luminal side of polarized epithelial cells. The lack of cellular polarity on malignant cells on the other hand, will lead to binding of FR $\alpha$ targeted agents through the whole tumor [18].

In conclusion, this study shows high concordance rates of $\mathrm{FR} \alpha$ expression between primary tumor and corresponding biopsy and metastatic tissue of both breast cancer and NSCLC patients, underscoring applicability of FR $\alpha$-targeted agents in these patients. Moreover, an association between FR $\alpha$ expression and histological/ molecular subtype of tumors was shown. Importantly, false positivity of FR $\alpha$ expression on biopsies was limited to less than $5 \%$. Although this study is performed with a relatively small amount of tissue specimens, the current results provide rational for the use of biopsies to select lung and breast cancer patients for FR $\alpha$-targeted agents, eventually leading to optimal personalized medicine.

\section{MATERIALS AND METHODS}

\section{Tissue samples}

The total cohort of NSCLC patients consisted of 38 patients who received curative surgery between 2011-2013 $(N=38)$ and 22 patients with distant metastases (20002013). Of all 60 NSCLC patients, 34 primary tumors containing adenocarcinoma and 26 SCCs were included as shown in Table 1. To assess the correlation between FR $\alpha$ expression on primary tumor and preoperatively obtained biopsy tissue, all available biopsy specimens, obtained via mediastinal biopsy, were collected. However, in some patients a transesophageal endoscopic ultrasound fine needle aspiration (EUS-FNA) or endobronchial ultrasound transbronchial needle aspiration (EBUSTBNA) was performed and some patients were referred to our academic center, of whom biopsy specimen could not be obtained. In total, 23 biopsies were collected: 12 biopsies containing adenocarcinoma and 11 biopsies containing SCC (Table 2). Moreover, 60 metastatic LNs of 33 patients were stained for FR $\alpha$. Adenocarcinoma was present in 42 of these LNs, while SCC was present in the remaining 18 metastatic LNs. Fifteen of the distant metastases contained adenocarcinoma, whereas 8 of them contained SCC. Of one patient who suffered from adenocarcinoma, tissues from two different metastatic sites were included. Metastases originated mostly from the brain $(n=10)$, followed by metastases from bone ( $n$ $=7)$, liver $(n=3)$, skin $(n=1)$, adrenal gland $(n=1)$ and jejunum $(n=1)$.

The total cohort of breast cancer patients consisted of 40 patients, who had undergone a breast conserving surgery (lumpectomy) for a cT1 or cT2 stage tumor between 2000-2014. In total, 6 of the 40 patients received neoadjuvant chemotherapy, of whom obtained results are separately described. The first cohort of breast cancer patients consisted of 20 patients of whom biopsy specimen and lumpectomy tissue was stained. In addition, a second cohort of 20 patients was included to assess concordance of FR $\alpha$ expression between biopsy specimen, lumpectomy and metastatic LNs. Of all patients, the receptor status of the tumor tissue was known, e.g. ER, PR and HER2 status.

\section{Immunohistochemistry}

IHC was performed using formalin-fixed, paraffinembedded (FFPE) tissue, obtained from the Pathology archive from the Leiden University Medical Center, 
Diakonessen Hospital and Rijnland Hospital. All samples were handled in an anonymous fashion according to the national ethical guidelines ('Code for Proper Secondary Use of Human Tissue,' Dutch Federation of Medical Scientific Societies) and approved by the Institutional Ethics Committee of the Leiden University Medical Center. Tissue samples were stained using the monoclonal antibody (Mab) 26B3.F2 (certified Folate Receptor alpha IHC Assay Kit, Biocare Medical) and subsequently scored for staining. The Mab26B3.F2 is highly specific for FR $\alpha$ without cross-reactivity to the other FR, e.g. FR $\beta$, FR $\gamma$ or $\mathrm{FR} \delta$. Validation of the staining protocol was performed by using (the recommended) lung adenocarcinoma as positive control and normal liver as negative control. Immunohistochemical staining was performed on 4 um paraffin sections on adhesive slides. Slides were deparaffinized in xylene and rehydrated in decreasing concentrations of ethanol. After rinsing in distilled water, endogenous peroxidase was blocked with hydrogen peroxide for five minutes. Slides were rinsed in water and antigen retrieval was performed in the DAKO PT Link, Target Retrieval Solution $\mathrm{pH} 6.0$ at $95^{\circ} \mathrm{C}$ for 10 minutes. After rinsing in PBS, nonspecific sites were blocked with a protein block (Background Punisher) for 5 minutes and the secondary antibody was incubated for 30 minutes on room temperature. As a negative control, one control slide was incubated with negative control reagent. After three washes the slides were incubated with MACH4 Mouse Probe Primary Antibody Enhancer (Biocare Medical) for 10 minutes. Again slides were washed in PBS, followed by incubation with MACH4 HRP Polymer for 10 minutes. Subsequently, the slides were washed and antibody binding was visualized by using 3,3'-diaminobenzidine (DAB, DAKO). Sections were counterstained with haematoxylin, dehydrated and mounted with pertex.

\section{Scoring method}

FR $\alpha$ expression was assessed in both lung and breast cancer tissue by using a membranous scoring method with a scale ranging from 0 to $3+$, as described by O'Shannessy et al. [14]. A score of 0 corresponded with absence of staining; 1+ equaled faint staining on luminal borders; 2+ equaled moderate staining on apical and sometimes lateral borders and $3+$ indicated strong circumferential staining (Figure 1). The tumor was considered positive when more than $10 \%$ of malignant cells were positively stained $(>0)$. Overexpression of FR $\alpha$ was defined as a score of $2+$ or $3+$. Homogeneity was described when $\mathrm{FR} \alpha$ expression was detectable on all malignant cells. Concordance was achieved when the expression score of one of the stained tissues, i.e. biopsy or corresponding LN or distant metastasis, matched the score of the primary tumor and both displayed either positive or negative expression of FR $\alpha$. In general, FR $\alpha$ expression of the primary tumor specimen was considered as golden standard. Concordance between either biopsy or metastatic tissue with the primary tumor was described as down- or upregulation of $\operatorname{FR} \alpha$. False positive expression was determined as positive FR $\alpha$ expression on biopsy or metastatic tissue without FR $\alpha$ expression on the primary tumor. Evaluation of the immunohistochemical staining was performed blinded independently by two observers. Disagreements were resolved by consensus after reviewing the relevant slide with the pathologist.

\section{Statistical analysis}

The statistical analysis was performed using SPSS version 22.0 software (SPSS, CIBM Corporation, Somer NY, USA). FR $\alpha$ expression per histopathological subgroup was calculated by the Fishers exact test. A P-value of $<$ 0.05 was considered statistically significant.

\section{ACKNOWLEDGMENTS}

The authors gratefully acknowledge R. Keyzer for his support and immunohistochemical expertise and Prof. Dr. V.T.H.B.M. Smit, pathologist, for his contribution to the analysis of the stained tissues. In addition, we would like to thank the research group at the University of Pennsylvania Perelman School of Medicine for providing us with an image of intraoperative FR $\alpha$-targeted NSCLC detection.

\section{CONFLICTS OF INTEREST}

None

\section{FUNDING}

This research was supported by the Dutch Cancer Society (grant UL2010-4732). This work was supported in part by the Nuts Ohra Fund.

\section{REFERENCES}

1. Maitland ML and Schilsky RL. Clinical trials in the era of personalized oncology. CA Cancer J Clin. 2011; 61:365381.

2. Kourea HP, Zolota V and Scopa CD. Targeted pathways in breast cancer: molecular and protein markers guiding therapeutic decisions. Curr Mol Pharmacol. 2014; 7:4-21.

3. Roengvoraphoj M, Tsongalis GJ, Dragnev KH and Rigas JR. Epidermal growth factor receptor tyrosine kinase inhibitors as initial therapy for non-small cell lung cancer: focus on epidermal growth factor receptor mutation testing and mutation-positive patients. Cancer Treat Rev. 2013; 39:839-850.

4. Coney LR, Tomassetti A, Carayannopoulos L, Frasca V, 
Kamen BA, Colnaghi MI and Zurawski VR, Jr. Cloning of a tumor-associated antigen: MOv18 and MOv19 antibodies recognize a folate-binding protein. Cancer Res. 1991; 51:6125-6132.

5. Ross JF, Chaudhuri PK and Ratnam M. Differential regulation of folate receptor isoforms in normal and malignant tissues in vivo and in established cell lines. Physiologic and clinical implications. Cancer. 1994; 73:2432-2443.

6. Della-Longa S and Arcovito A. Structural and functional insights on folate receptor alpha (FRalpha) by homology modeling, ligand docking and molecular dynamics. J Mol Graph Model. 2013; 44:197-207.

7. Elnakat $\mathrm{H}$ and Ratnam M. Distribution, functionality and gene regulation of folate receptor isoforms: implications in targeted therapy. Adv Drug Deliv Rev. 2004; 56:10671084.

8. Gonen N and Assaraf YG. Antifolates in cancer therapy: structure, activity and mechanisms of drug resistance. Drug Resist Updat. 2012; 15:183-210.

9. Weitman SD, Lark RH, Coney LR, Fort DW, Frasca V, Zurawski VR, Jr. and Kamen BA. Distribution of the folate receptor GP38 in normal and malignant cell lines and tissues. Cancer Res. 1992; 52:3396-3401.

10. Assaraf YG, Leamon CP and Reddy JA. The folate receptor as a rational therapeutic target for personalized cancer treatment. Drug Resist Updat. 2014; 17:89-95.

11. de GP and Munden RF. Lung cancer epidemiology, risk factors, and prevention. Radiol Clin North Am. 2012; 50:863-876.

12. Gadgeel SM, Ramalingam SS and Kalemkerian GP. Treatment of lung cancer. Radiol Clin North Am. 2012; 50:961-974.

13. Nunez MI, Behrens C, Woods DM, Lin H, Suraokar M, Kadara H, Hofstetter W, Kalhor N, Lee JJ, Franklin W, Stewart DJ and Wistuba II. High expression of folate receptor alpha in lung cancer correlates with adenocarcinoma histology and EGFR [corrected] mutation. J Thorac Oncol. 2012; 7:833-840.

14. O'Shannessy DJ, Yu G, Smale R, Fu YS, Singhal S, Thiel RP, Somers EB and Vachani A. Folate receptor alpha expression in lung cancer: diagnostic and prognostic significance. Oncotarget. 2012; 3:414-425. doi: 10.18632/ oncotarget.489.

15. Chen L, Linden HM, Anderson BO and Li CI. Trends in 5-year survival rates among breast cancer patients by hormone receptor status and stage. Breast Cancer Res Treat. 2014; 147:609-616.

16. O'Shannessy DJ, Somers EB, Maltzman J, Smale R and $\mathrm{Fu}$ YS. Folate receptor alpha (FRA) expression in breast cancer: identification of a new molecular subtype and association with triple negative disease. Springerplus. 2012; $1: 22$.

17. Sega EI and Low PS. Tumor detection using folate receptor- targeted imaging agents. Cancer Metastasis Rev. 2008; 27:655-664.

18. Low PS and Kularatne SA. Folate-targeted therapeutic and imaging agents for cancer. Curr Opin Chem Biol. 2009; 13:256-262.

19. Matherly LH and Hou Z. Structure and function of the reduced folate carrier a paradigm of a major facilitator superfamily mammalian nutrient transporter. Vitam Horm. 2008; 79:145-184.

20. Hilgenbrink AR and Low PS. Folate receptor-mediated drug targeting: from therapeutics to diagnostics. J Pharm Sci. 2005; 94:2135-2146.

21. Shi H, Guo J, Li C and Wang Z. A current review of folate receptor alpha as a potential tumor target in non-small-cell lung cancer. Drug Des Devel Ther. 2015; 9:4989-4996.

22. Okusanya OT, DeJesus EM, Jiang JX, Judy RP, Venegas OG, Deshpande CG, Heitjan DF, Nie S, Low PS and Singhal S. Intraoperative molecular imaging can identify lung adenocarcinomas during pulmonary resection. J Thorac Cardiovasc Surg. 2015; 150:28-35.

23. Kyndi M, Sorensen FB, Knudsen H, Overgaard M, Nielsen HM, Andersen J and Overgaard J. Tissue microarrays compared with whole sections and biochemical analyses. A subgroup analysis of DBCG 82 b\&c. Acta Oncol. 2008; 47:591-599.

24. Bremer RE, Scoggin TS, Somers EB, O'Shannessy DJ and Tacha DE. Interobserver agreement and assay reproducibility of folate receptor alpha expression in lung adenocarcinoma: a prognostic marker and potential therapeutic target. Arch Pathol Lab Med. 2013; 137:17471752.

25. Sutherland KD and Berns A. Cell of origin of lung cancer. Mol Oncol. 2010; 4:397-403.

26. Cagle PT, Zhai QJ, Murphy L and Low PS. Folate receptor in adenocarcinoma and squamous cell carcinoma of the lung: potential target for folate-linked therapeutic agents. Arch Pathol Lab Med. 2013; 137:241-244.

27. Hartmann LC, Keeney GL, Lingle WL, Christianson TJ, Varghese B, Hillman D, Oberg AL and Low PS. Folate receptor overexpression is associated with poor outcome in breast cancer. Int J Cancer. 2007; 121:938-942.

28. Zhang Z, Wang J, Tacha DE, Li P, Bremer RE, Chen H, Wei B, Xiao X, Da J, Skinner K, Hicks DG, Bu H and Tang P. Folate receptor alpha associated with triple-negative breast cancer and poor prognosis. Arch Pathol Lab Med. 2014; 138:890-895.

29. Leone JP, Bhargava R, Theisen BK, Hamilton RL, Lee AV and Brufsky AM. Expression of high affinity folate receptor in breast cancer brain metastasis. Oncotarget. 2015; 6:30327-30333. doi: 10.18632/oncotarget.4639.

30. Iwakiri S, Sonobe M, Nagai S, Hirata T, Wada H and Miyahara R. Expression status of folate receptor alpha is significantly correlated with prognosis in non-small-cell lung cancers. Ann Surg Oncol. 2008; 15:889-899. 
31. Rochman H, Selhub J and Karrison T. Folate binding protein and the estrogen receptor in breast cancer. Cancer Detect Prev. 1985; 8:71-75.

32. Kelley KM, Rowan BG and Ratnam M. Modulation of the folate receptor alpha gene by the estrogen receptor: mechanism and implications in tumor targeting. Cancer Res. 2003; 63:2820-2828.

33. Kikuchi $\mathrm{T}$, Daigo $\mathrm{Y}$, Ishikawa N, Katagiri $\mathrm{T}$, Tsunoda T, Yoshida S and Nakamura Y. Expression profiles of metastatic brain tumor from lung adenocarcinomas on cDNA microarray. Int J Oncol. 2006; 28:799-805.
34. van Driel PB, van de Giessen M, Boonstra MC, Snoeks TJ, Keereweer S, Oliveira S, van de Velde CJ, Lelieveldt BP, Vahrmeijer AL, Lowik CW and Dijkstra J. Characterization and evaluation of the artemis camera for fluorescenceguided cancer surgery. Mol Imaging Biol. 2015; 17:413423. 